\title{
Team-Based Health Information Exchange Use Increased Mammography Documentation and Referral in an Academic Primary Care Practice: An Interrupted Time Series
}

\author{
Samantha I. Pitts, MD, MPH ${ }^{7}$, Nisa M. Maruthur, $M D, M H S^{7}$, Xiao Wang, $B S^{2}$, \\ Melinda D. Sawyer, MSN, RN, CSN-BC ${ }^{3}$, Renee Grimes' , Candace Nigrin', \\ Jeanne M. Clark, MD, MPH' , Nae-Yuh Wang, $P h D^{7}$, Heather F. Sateia, MD ${ }^{7}$, and Kimberly S. Peairs, \\ $M D^{7}$
}

'School of Medicine, Division of General Internal Medicine, Johns Hopkins University, Baltimore, MD, USA; ${ }^{2}$ School of Medicine, Johns Hopkins University, Baltimore, MD, USA; ${ }^{3}$ Johns Hopkins Armstrong Institute for Patient Safety and Quality, Baltimore, MD, USA.

\begin{abstract}
BACKGROUND: Few studies have examined the impact of health information exchanges (HIE) on quality in ambulatory settings.

METHODS: From September 29, 2014, to September 4, 2015, we conducted an interrupted time series analysis of query-based use of the state HIE as part of team-based care to improve mammography screening in an academic primary care practice. Women aged 50-74 years with a practice visit and who were eligible for mammography were included. We conducted non-parametric data analysis using LOESS, followed by ARIMA analysis.
\end{abstract}

RESULTS: During the study period, there were 2020 visits among 904 eligible patients, including 648 visits among 485 patients during 16 baseline weeks, and 1372 visits by 755 patients during 33 intervention weeks. During the intervention period, $16.0 \%$ of eligible women who were not up to date in our EHR had a mammogram in the HIE. Of eligible women, the proportion who had a documented up-to-date mammogram at the time of their visit increased by $11.3 \%$, from $73.4 \%$ at baseline to $84.7 \%$ ( $p<$ $0.0001)$, the proportion who had mammography addressed at the time of their visit increased by $42.7 \%$, from $32.7 \%$ at baseline to $75.4 \%(p<0.0001)$, and the proportion who were up to date at 8 weeks post-visit increased by $11.7 \%$, from $76.3 \%$ at baseline to $88.0 \%$ ( $p<0.0001)$.

DISCUSSION: Query-based use of the state HIE as part of team-based care improved documentation of mammography and led to an increase in the proportion of eligible women who received counseling on mammography screening in one primary care practice. These results suggest that HIE use in primary care could lead to improved delivery of other preventive services.

KEY WORDS: health information exchange; primary care; preventive health services; mammography.

Electronic supplementary material The online version of this article (https://doi.org/10.1007/s11606-017-4259-8) contains supplementary material, which is available to authorized users.

Received February 21, 2017

Revised July 14, 2017

Accepted December 1, 2017

Published online February 1, 2018
J Gen Intern Med 33(5):710-4

DOI: $10.1007 /$ s11606-017-4259-8

(c) Society of General Internal Medicine 2018

\section{INTRODUCTION}

The enactment of the Health Information Technology for Economic and Clinical Health (HITECH) Act in 2009 was followed by rapid adoption of health information technology and development of health information exchange (HIE) infrastructure nationwide with the support of the state HIE Program. ${ }^{1}$ By 2013, all 50 states had established HIEs, ${ }^{2}$ and in $2015,78 \%$ of medical offices were estimated to have implemented a certified electronic health record (EHR). ${ }^{3}$ Although providers are often missing critical medical data, ${ }^{4}$ electronic exchange of information by ambulatory practices remains low; only about one-third of medical offices reported sending and/ or accessing electronic records from other providers in $2015 .^{5}$

Barriers to health information exchange may contribute to this limited use in outpatient settings. Frequently identified barriers include perceived inadequacy or incompleteness of information within the HIE and the impact on workflow. ${ }^{6}$ In addition, few studies have examined the impact of HIE on quality in ambulatory care settings; further understanding of the benefits could present a compelling case for increased utilization.

We sought to improve the documentation of and referral for screening mammography for women at the time of a clinical visit through 1) development of a team-based clinical workflow to standardize practice procedures, and 2) incorporation of query-based use of the state HIE to identify women with mammograms not included in our EHR. Although the U.S. Preventive Services Task Force recommends screening mammography every 2 years for women 50-74 years of age, in 2015 only $71.5 \%$ of eligible women nationally reported screening within the past 2 years, with lower rates among those with lower education and income levels, leading to missed opportunities for detecting and treating breast cancer. ${ }^{7}$ Within the Johns Hopkins Medical Alliance for Patients, an 
accountable care organization (ACO), in 2014, only $60.4 \%$ of eligible patients had documented screening mammography. ${ }^{8}$ However, as women may obtain primary care at one location and subspecialty care, including gynecology, at another, mammography results may not be available within the Johns Hopkins EHR. We hypothesized that use of the HIE would result in more complete data on receipt of preventive care services, and that complete mammography data could enable screening efforts targeted toward women overdue for mammography.

\section{METHODS}

Context. Johns Hopkins Green Spring General Internal Medicine (GIM) is an academic suburban general medical practice within the Johns Hopkins ACO, which has used the Epic software system (Epic Systems Corporation, Verona, WI) since 2013. There are 16 faculty and general internal medicine fellows in the practice, for a total of six full-time-equivalent providers. This practice has a relatively stable patient population, with approximately 7000 patient visits recorded in 2014; among visits in 2015 and 2016, fully $90 \%$ were return appointments and $10 \%$ new patients. In October 2014, Green Spring GIM implemented the Comprehensive Unit-based Safety Program (CUSP), which provides training in systems of care delivery and structured processes to identify and address patient safety and quality concerns. ${ }^{9}$

Intervention. A representative multidisciplinary team of staff members engaged in CUSP at Green Spring GIM used the Translating Evidence into Practice framework ${ }^{10}$ to identify gaps in existing processes and factors contributing to incomplete mammography screening of our patients. From this process, the team recognized multiple barriers to care (E-Figure 1), which included missing data, as our EHR lacked the results of mammograms that were obtained outside the Johns Hopkins Health System, and a lack of explicit policies and standard procedures. Because preliminary data indicated we had missed opportunities to discuss mammography screening in person, and because we believed that complete data would be essential to effective patient outreach, our team decided to begin by improving processes within the practice visit. The team developed and implemented an intervention which incorporated a query of the designated HIE for the state of Maryland, the Chesapeake Regional Information System for our Patients, or CRISP, ${ }^{11}$ into the staff workflow to identify mammograms completed outside our health system and to standardize mammography documentation and referral (E-Figure 2).

Women aged 50-74 years with a visit to Green Spring GIM during the period from January 19, 2015, to September 4, 2015 , were eligible for the protocol. Women who were not candidates for mammography (e.g., history of bilateral mastectomy) as previously indicated by clinicians within the health maintenance section were excluded. We defined women as "up to date on mammography" if they had a screening or diagnostic mammogram report within the prior 24 months in the EHR.

An office coordinator manually reviewed the schedule to identify women who were not up to date on mammography, using the mammography health care maintenance field of the EHR. For these women, the office coordinator queried CRISP for mammography completed outside our health system within the past 24 months; if found, the mammogram was scanned into the patient's medical record. Women who remained overdue for mammography were flagged by the office coordinator for review by the medical assistant (MA) at the time of the appointment. At the visit, if a prior physician order was present, the MA reminded the patient to obtain the mammogram. Otherwise, the MA placed an order for review and signature by the provider. If the patient refused or identified a reason that mammography was not indicated, the MA notified the provider through the EHR to update the health care maintenance section. If the patient reported an outside mammogram that was not identified by review of CRISP, the medical office coordinator was notified; the office coordinator would then request the results directly from the outside facility, which were scanned and routed to the physician when available. All instructions from the MA to the patient were documented using standardized text in the after-visit summary for review by the visit provider.

Following 16 weeks beginning September 29, 2014 that served as baseline, pilot implementation began January 19, 2015 (during weeks 17-19), when we experimented with the frequency of HIE query. We settled on a daily workflow to capture patients scheduled for acute appointments and to reduce waste due to cancellations. During weeks 20-22, we obtained feedback from clinicians and staff on the revised protocol following pilot testing. We implemented the daily workflow in week 23 with a limited number of clinicians, and expanded to all clinicians by week 27 .

Analysis. We conducted an interrupted time series analysis to evaluate the impact of our intervention on four measures among eligible women: 1) the proportion of eligible women who had their records updated through the use of the HIE; 2) the proportion who were not up to date on mammography at the time of their visit; 3) the proportion who had mammography addressed at their visit; and 4) the proportion who were up to date on mammography at 8 weeks following their visit. We considered that mammography was "addressed at the time of a visit" if there was documented discussion of mammography in the encounter record (including refusal), or an order for mammography was placed.

We used Epic Reporting Workbench to identify the most recent screening mammogram within the Johns Hopkins system among eligible women during the 49 weeks from September 29, 2014, to September 4, 2015, including 16 baseline weeks and 33 intervention weeks, and conducted medical record review to evaluate the impact of this protocol. For 
women who did not have an up-to-date mammogram in the Reporting Workbench report, study team members used standardized procedures to abstract data from health care maintenance, imaging, and outside media to capture diagnostic mammograms and any mammogram performed outside the Johns Hopkins system. Medical records were abstracted by one reviewer and verified by a second reviewer. The medical office coordinator recorded the number of mammograms obtained each week of the intervention period through the HIE. Data were aggregated to assess weekly performance. We conducted non-parametric data analysis using locally weighted scatterplot smoothing (LOESS) to estimate patterns of weekly outcome trajectories over time, followed by interrupted time series analyses using autoregressive integrated moving average (ARIMA) models to evaluate specific period effects in the time series, both using SAS software (version 9.4; SAS Institute Inc., Cary, NC). In ARIMA analysis for each outcome, we modeled two segments of the intervention (rollout in weeks 17-26 and full implementation beginning in week 27) to assess for a potential change in effect of the intervention over time based on the LOESS models. As a stable effect was seen from week 27 to week 49 , without change over time, we present the comparison of the baseline and intervention time periods, consistent with our a priori hypotheses.

This study was reviewed by the Johns Hopkins School of Medicine Institutional Review Board and acknowledged as a quality improvement project.

\section{RESULTS}

During the study period, there were 2020 visits by 904 eligible patients [763 (84\%) with $1-3$ visits, 122 (13\%) with 4-6 visits, 19 (2\%) with $7-11$ visits], including 648 visits by 485 patients during the 16 baseline weeks and 1372 visits by 755 patients during the 33 intervention weeks. The median number of eligible patients per week was 44 (range 16-61). The median age of patients at the time of visit was 62 years. During the intervention period, 31 patients who were overdue for mammography had their EHR reviewed more than once [twice: 22 (71\%), 3 times: 8 (26\%), 4 times: 1(3\%)].

During the intervention period, 65 of $755(8.6 \%)$ eligible women had a relevant mammogram identified in the HIE (Table 1), including $16.0 \%$ of women who were categorized as not up to date on mammography based on records in the Johns Hopkins EHR. The weekly proportion of women who were not up to date in our EHR with a mammogram identified in the HIE is presented in E-Fig. 3.

During the intervention period, the proportion of eligible women who had a documented up-to-date mammogram at the time of their visit increased by 11.3 percentage points, from $73.4 \%$ to $84.7 \%$ ( $p<0.0001$, E-Figure 4$)$, the proportion who had mammography addressed at the time of their visit increased by 42.7 percentage points, from $32.7 \%$ to $75.4 \%$
Table 1 Summary of Mammography Outcomes at Baseline and during Implementation

\begin{tabular}{|c|c|c|}
\hline \multirow[t]{2}{*}{ Outcome } & \multirow{2}{*}{$\begin{array}{l}\text { Baseline } \\
648 \text { visits } \\
485 \text { patients }\end{array}$} & \multirow{2}{*}{$\begin{array}{l}\begin{array}{l}\text { Implementation } \\
\text { phase }\end{array} \\
1372 \text { visits } \\
755 \text { patients }\end{array}$} \\
\hline & & \\
\hline $\begin{array}{l}\text { Mammogram updated } \\
\text { through HIE }\end{array}$ & - & $8.6 \%$ of patients \\
\hline $\begin{array}{l}\text { Mammogram up to date at } \\
\text { visit }\end{array}$ & $73.4 \%$ of visits & $84.7 \%$ of visits \\
\hline $\begin{array}{l}\text { Mammogram addressed at } \\
\text { visit }\end{array}$ & $32.7 \%$ of visits & $75.4 \%$ of visits \\
\hline $\begin{array}{l}\text { Mammogram up to date } 8 \\
\text { weeks after visit }\end{array}$ & $76.3 \%$ of visits & $88.0 \%$ of visits \\
\hline $\begin{array}{l}\text { Mammogram newly obtained } \\
\text { by } 8 \text { weeks after clinic visit }\end{array}$ & $2.9 \%$ of visits & $3.3 \%$ of visits \\
\hline
\end{tabular}

$(p<0.0001)$, and the proportion who were up to date at 8 weeks following their visit increased by 11.7 percentage points, from $76.3 \%$ to $88.0 \%(p<0.0001)$. In each case, the increase was sustained across the duration of the intervention.

\section{DISCUSSION}

We created a standardized, daily workflow for query of the state HIE to identify mammograms performed outside our health system. This resulted in an 11-percentage-point increase in the proportion of our patients who were documented within our EHR as being up to date on mammography. More complete mammography data allowed our practice to identify with greater certainty those women who were overdue and to routinely offer mammography screening to women who were not up to date; during the intervention period, our multidisciplinary team addressed mammography in three-quarters of visits with women who were overdue for mammography.

Our study demonstrates the potential value of the incorporation of HIE data and team-based care for preventive care services. A systematic review from 2014 concluded that, based on Grading of Recommendations Assessment, Development and Evaluation (GRADE) criteria, ${ }^{12}$ there is lowquality evidence for the impact of HIEs on utilization or cost in emergency departments. ${ }^{13}$ Most studies examined HIE use in inpatient or emergency department settings; only two studies were identified in ambulatory care. ${ }^{14,15}$ A retrospective cohort study of providers within a regional independent practice association found that use of a regional HIE was associated with improved performance on a composite quality measure in multivariable analysis (odds ratio 1.42 , confidence interval 1.04-1.95). In bivariate analysis, providers who used the HIE were more likely to exceed the regional benchmark on mammography $(57.6 \%$ vs. $34.6 \%, p=0.01) .{ }^{14}$ A claims analysis of a regional HIE found that HIE adoption was associated with small decreases in laboratory, but not radiology, test 
orders. ${ }^{15}$ No studies were identified that assessed a standardized protocol for HIE use in ambulatory care.

Studies of HIE generally indicate that use occurs in a small proportion of encounters. ${ }^{13}$ Although we did not formally track the proportion of encounters in which the HIE was queried, implementation of a standardized workflow, such as ours, for integration of data from the HIE ensures a higher level of use than has been demonstrated in prior studies.

Despite our standardized, team-based model of care, we were not able to address mammography with all women who were overdue at the time of their clinic visit. A systematic examination of our failures indicated that failure to address overdue mammography occurred primarily at times of peak workflow for the MA or when patients were late.

With alternative payment models, including ACOs and the Medicare Quality Payment Program, health care providers and health systems are held to greater accountability standards for the health of patients, including metrics for delivery of preventive care. Thus, these systems are incentivized to implement population health initiatives, such as patient outreach, to improve these metrics. Complete data are essential for accurate identification and targeted messaging to patients who are due for preventive care services.

Our intervention was successful in part because all major radiology providers performing mammography in our geographic area had participated in the HIE longer than the 2year interval for mammography screening. Thus we avoided a major barrier that had been identified previously - a lack of data within the HIE. ${ }^{6}$ In addition, patients must actively opt out of CRISP; as a result, it may have more complete data than other state HIEs. In future work, we plan to extend this intervention to other key preventive services, such as immunizations, pap smear, and colonoscopy. While much current work with HIE rightfully involves communication surrounding care transitions, the incorporation of data from ambulatory care sites, such as colonoscopy, and linkage to immunization information system data are needed to assess the potential impact of HIE on the delivery of preventive care.

We created a standard workflow with a designated nonclinician staff member to query the HIE; this reduced the impact on clinic throughput and provider workflow that has been a concern in other studies. Our protocol required staff time not only for the query but also for manual scanning of mammograms into our EHR. Improved interoperability and integration of HIE data directly into the EHR would significantly reduce the staff time required and would allow for broader incorporation of HIE data in primary care practices. With better integration of HIE data into the EHR, and/or tools to more readily obtain data on populations of patients, data from HIEs could be used to identify and address preventive care in all practice patients who need screening. Johns Hopkins Medicine is currently working to integrate data from CRISP into the EHR.

This study is the first to document the impact of a querybased workflow for HIE use to improve the delivery of preventive services within primary care, and featured a strong observational study design. However, our study is limited by the inclusion of only one practice and the lack of more comprehensive demographic data on patient participants; the proportion of patients with data outside the health system where they receive primary care will likely vary based on the setting and the demographics of the population. Additionally, we included all women aged 50-74 years who were eligible for mammography in our target population; as a result, our definition of up to date on mammography would be insufficient for some women undergoing the procedure for breast cancer surveillance. Further studies are needed to assess the use of HIE for preventive care in other practice settings.

Our intervention is limited in that it addressed mammography screening only for women who presented for care. Women who did not present to the practice may be at higher risk for advanced breast cancer. However, we felt this was a critical first step toward improving screening for our patients, as each visit in which we did not address mammography was a missed opportunity. Our protocol likely increased clinician efficiency; by obtaining outside mammograms prior to the patients' visits and including the MA in the process, clinicians were able to focus conversations about screening on the appropriate patients. Although patients described here were required to have a clinic visit, we now address mammography for women who present for urgent care as well as primary care visits. Our standardized, team-based protocol and more complete data now serve as a foundation for our next steps, which include reducing barriers to screening in order to improve completion among women referred to mammography and providing outreach to women who are not engaged in care.

\section{CONCLUSION}

Query-based use of the state HIE as part of team-based care improved documentation of mammography and led to an increase in the proportion of overdue women who received counseling on mammography screening in one primary care practice. These results suggest that HIE use in primary care could lead to improved delivery of other preventive services for which data are available. Additional studies are needed to confirm these results in other practice settings.

Acknowledgements: We would like to acknowledge Crystal Ki, MBA, MPH, for her assistance with data abstraction.

Corresponding Author: Samantha I. Pitts, MD, MPH; School of Medicine, Division of General Internal Medicine Johns Hopkins University, Baltimore, MD, USA (e-mail: spitts4@jhmi.edu).

Funders This publication was made possible by a grant from the Johns Hopkins Primary Care Consortium and by the Johns Hopkins Institute for Clinical and Translational Research (ICTR), which is funded in part by grant no. KL2TR001077 from the National Center 
for Advancing Translational Sciences (NCATS), a component of the National Institutes of Health (NIH), and NIH Roadmap for Medical Research. Its contents are solely the responsibility of the authors and do not necessarily represent the official view of the Johns Hopkins ICTR, NCATS, or NIH.

Prior presentations The work in this manuscript was previously presented at The Johns Hopkins Patient Safety Summit, Baltimore, Maryland, October 2, 2015 and The Society for General Internal Medicine Annual Meeting, Hollywood, Florida, May 13, 2016.

\section{Compliance with Ethical Standards:}

Conflict of Interest: The authors declare that they do not have a conflict of interest.

\section{REFERENCES}

1. Office of the National Coordinator for Health Information Technology. 2016 Report to Congress on Health IT Progress. Washington, D.C.: Department of Health and Human Services; 2016.

2. State HIE Implementation Status. Washington, D.C.: 2014. Office of the National Coordinator for Health IT. Available at: https://www.healthit. gov/policy-researchers-implementers/state-hie-implementation-status). Accessed November 8, 2017.

3. Jamoom E. YN. Table of Electronic Health Record Adoption and Use among Office-based Physicians in the U.S., by State: 2015 National Electronic Health Records Survey. Hyattsville: National Center for Health Statistics; 2016.

4. Smith PC, Araya-Guerra R, Bublitz C, et al. Missing clinical information during primary care visits. JAMA. 2005;293:565-71.

5. National Center for Health Statistics. State Variation in Electronic Sharing of Information in Physicians Offices: United States, 2015. NCHS
Data brief No. 261. Hyattsville: National Center for Health Statistics; 2016.

6. Eden KB, Totten AM, Kassakian SZ, et al. Barriers and facilitators to exchanging health information: a systematic review. Int J Med Inform. 2016;88:44-51.

7. Sabatino SA, White MC, Thompson TD, Klabunde CN, Centers for Disease Control and Prevention (CDC). Cancer screening test use United States, 2015. MMWR Morb Mortal Wkly Rep. 2017;66(8);201-6.

8. Medicare Shared Savings Accountable Care Organizations Performance Year 2014 Results. Baltimore, Maryland: 2015. Centers for Medicare and Medicaid Services. Available at: https://data.cms.gov/ACO/MedicareShared-Savings-Program-Accountable-Care-O/ucce-hhpu). Accessed November 8, 2017.

9. Pronovost P, Weast B, Rosenstein B, et al. Implementing and Validating a Comprehensive Unit-Based Safety Program. J Patient Saf. 2005;1:3340.

10. Pronovost PJ, Berenholtz SM, Needham DM. Translating Evidence into Practice: a model for large scale knowledge translation. BMJ. 2008; 337:a1714.

11. Chesapeake Regional Information System for our Patients (CRISP). Columbia, MD: Date unknown. CRISP. Available at: http://crisphealth. org). Accessed November 8, 2017

12. Guyatt GH, Oxman AD, Vist GE, Kunz R, Falck-Ytter Y, Alonso-Coello P, GRADE Working Group et al.. GRADE: an emerging consensus on rating quality of evidence and strength of recommendations. BMJ. 2008;336:924-6.

13. Rudin RS, Motala A, Goldzweig CL, Shekelle PG. Usage and effect of health information exchange: a systematic review. Ann Intern Med. 2014;161:803-11.

14. Kern LM, Barron Y, Dhopeshwarkar RV, Kaushal R. Health information exchange and ambulatory quality of care. Appl Clin Inform. 2012;3:197209.

15. Ross SE, Radcliff TA, Leblanc WG, Dickinson LM, Libby AM, Nease DE Jr. Effects of health information exchange adoption on ambulatory testing rates. J Am Med Inform Assoc. 2013;20:1137-42. 